\title{
异乳糖的合成研究
}

\author{
姜 锐 梁晓梅金淑慧张建军* 王道全 \\ (中国农业大学理学院应化系 北京 100193)
}

\begin{abstract}
摘要 异乳糖是一种还原性双糖, 在细菌的代谢过程中有着非常重要的生物学作用. 以 $D$-葡萄糖、 $D$-半乳糖为起始原 料, 探索了异乳糖的多羟基区域选择性偶联与非选择性偶联两条合成路线, 其中非选择性偶联路线经五锅反应，总收 率高达 $44 \%$, 中间体及目标产物易于分离纯化, 适宜于异乳糖的大量制备. 中间体及最终产物的结构均通过 NMR, HRMS 进行表征.
\end{abstract}

关键词 异乳糖; $D$-葡萄糖; $D$-半乳糖; 合成

\section{Efficient Synthesis of Allolactose}

\author{
Jiang, Rui Liang, Xiaomei Jin, Shuhui Zhang, Jianjun* Wang, Daoquan \\ (Key Laboratory of Pesticide Chemistry and Application Technology, Department of Applied Chemistry, \\ China Agricultural University, Beijing 100193)
}

\begin{abstract}
Allolactose $(\beta$ - $D$-galactopyranosyl- $(1 \rightarrow 6)-\alpha, \beta$ - $D$-glucopyranose $)$ is a reducing disaccharide playing important biological role in bacterial metabolism. Two synthetic methods of allolactose including regioselective glycosylation and stepwise synthesis were investigated using $D$-glucose and $D$-galactose as the starting materials. The later approach involved a five-spot strategy, $44 \%$ overall yields and all the intermediates were easily to be purified, making the route suitable for large scale preparation of the target compound. All the compounds were characterized by NMR and HRMS spectra.
\end{abstract}

Keywords allolactose; $D$-glucose; $D$-galactose; synthesis

异乳糖(1)是乳糖的异构体, 化学结构为半乳糖- $(\beta-$ $1 \rightarrow 6)$ - 葡萄糖, 是一种还原性双糖. 在 $\beta$-半乳糖苷酶的 作用下乳糖可以转变成异乳糖, 异乳糖是乳糖操纵子的 诱导物, 在细菌的代谢过程中有着非常重要的生物学作 用 ${ }^{[1]}$. 例如, 在大肠杆菌中异乳糖参与调节乳糖操纵 子 $^{[1,2]}$ 的活性, 乳糖操纵子包含 3 种结构基因 ( $L a c Z, L a c Y$ 及 $L a c A)$, 主要负责吸收和水解乳糖而作为大肠杆菌生 命活动的碳源. 如果在细菌生长环境中缺乏乳糖, 阻遏 蛋白会紧密地与位于启动子下游接近 $L a c Z$ 的短 DNA 序 列(称为乳糖操纵基因)结合. 与操纵基因结合的阻遏蛋 白会干扰 RNA 聚合酶与启动子的结合, 所以 mRNA 可 以在很低水平的情况下为 $L a c \mathrm{Z}$ 及 $L a c \mathrm{Y}$ 编码. 当细胞在 有乳糖的环境下成长时, 在代谢过程中乳糖被 $\beta$-半乳糖 苷酶催化水解产生异乳糖(Allolactose, Scheme 1), 异乳 糖能和调节基因产生的阻遏蛋白结合, 使阻遏蛋白改变 构象, 不能在和操纵基因结合, 失去阻遏作用, 结果
RNA 聚合酶便与启动基因结合，并使结构基因活化，转 录出 mRNA, 翻译出酶蛋白.

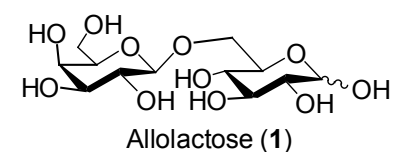

Scheme 1

由于异乳糖具有重要的生物学作用，其合成工作一 直受到人们的关注. 文献中已经报道的获得异乳糖的方 法主要有两种: 其一为化学合成, 通过制备澳代、氯代 半乳糖供体与六位为羟基的葡萄糖受体经偶联得到 ${ }^{[3]}$, 或者通过结构相近的天然产物经一系列化学反应转化 得到异乳糖, 例如 2003 年 Riva 课题组 ${ }^{[4]}$ 以天然产物 Amygdalin 为起始原料, 经过化学反应将非还原端葡萄 糖环上 4 位平伏键美基转换为直立建羟基, 从而完成异 乳糖的合成; 其二是酶合成方法, 即模仿异乳糖的天然

* E-mail: zhangjianjun@cau.edu.cn

Received December 22, 2013; revised January 13, 2014; published online February 10, 2014.

Project supported by the National Natural Science Foundation of China (No. 21172257) and the National S\&T Pillar Program of China (Nos. 2012BAK25B03-01, 2011AA10A206).

国家自然科学基金(No. 21172257)和十二五科技支撑计划(Nos. 2012BAK25B03-01, 2011AA10A206)资助项目. 
合成法一一利用 $\beta$-半乳糖苷酶催化水解乳糖而得到异 乳糖 ${ }^{[5]}$. 在一项合作研究中, 课题组需要获得十克级别 的异乳糖进行生物学研究, 但研究发现采用文献报道的 方法都因路线长、收率低、重现性不好、分离纯化困难 等因素而难以进行大量制备. 由于异乳糖的重要性, 寻 找成本低廉、操作简单、收率良好的异乳糖合成方法具 有十分重要的意义.

\section{1 结果与讨论}

以不保护或少保护的多羟基糖基为受体偶联进行 寡糖的构建, 由于步骤较少, 合成相对简洁而受到人们 广泛的关注 ${ }^{\left[{ }^{[} 8\right]}$, 本课题组在葟糖的区域选择性偶联方 面也进行了相关的研究 ${ }^{[9,10]}$. 因此在异乳糖的合成探索 中, 我们首先进行了其区域、立体选择性合成方法研究. 如 Scheme 2 所示, $D$-半乳糖(2)经全苯甲酰化、选择性脱 除异头苯甲酰基、三氯乙酰亚胺酯活化三步反应, 通过 优化反应条件, 可一锅法以 $84 \%$ 的产率得到单糖供体 $(3)^{[11]}$. 另一方面, 按文献方法 ${ }^{[12]}$ 以 $D$-葡萄糖(4)为原料 合成 1,2:5,6-O-双异丙叉- $\alpha$ - $D$-㫙喃葡萄糖(5). 化合物 $\mathbf{5}$ 在乙酸䣶、吡啶作用下 3 位羟基乙酰化, 蒸出溶剂后直 接以 $70 \%$ 乙酸、 $75{ }^{\circ} \mathrm{C}$ 条件下水解, 5,6- $O$ - 异丙叉基被选 择性脱除，1,2- $O$-异丙叉基不受影响. 两步反应以 $91 \%$
的收率得到 1,2- $O$-异丙叉-3- $O$-乙酰基- $\alpha$ - $D$-呋喃葡萄糖 (6). 以上三步也可以连续操作, 即以 $D$-葡萄糖(4)为起 始原料, 中间体 5 不经过柱层析纯化，可以 $82 \%$ 的收率 获得双羟基受体 6 .

双羟基受体 $\mathbf{6}$ 与供体 $\mathbf{3}$ 在 TMSOTf 催化下进行偶联 反应 ${ }^{[13]}$, 期待在受体 6 位选择性形成 $\beta-1 \rightarrow 6$ 连接糖苷 键. 但实验中发现反应得到并非单一产物. 将所得混合 产物分离纯化后得到目标 $\beta-1 \rightarrow 6$ 连接的产物 9 , 收率为 $81 \%, \beta-1 \rightarrow 5$ 连接的化合物 7, 收率为 $8 \%$. 其结构通过 游离羟基的乙酰化进行确定(图 1): $\beta-1 \rightarrow 5$ 连接的产物 7 的 H-6 的化学位移为 $\delta 3.71 \sim 3.91$, 其乙酰化所得化合 物 8 的 H-6 向低场移动, 化学位移为 $\delta 3.97,4.38$ 证明化 合物 7 的乙酰化反应发生在 6 位羟基上; $\beta-1 \rightarrow 6$ 连接的 双糖 9 的 H-5 的化学位移为 $\delta 3.82$, 其乙酰化反应所得 化合物 10 的 H-5 化学位移向低场移动到 $\delta 5.19$, 证明乙 酰化反应发生在化合物 9 的 5 位差基上.

此路线虽然简便，但由于反应所得并非单一产物， 目标产物 9 与副产物 7 极性相似, 大量制备时分离纯化 极为困难. 该方法在目标产物的小量合成时是行之有效 的, 但在异乳糖的大量合成中, 要获得足够纯度的目标 产物则需要反复硅胶柱纯化分离, 操作繁琐, 使得此路
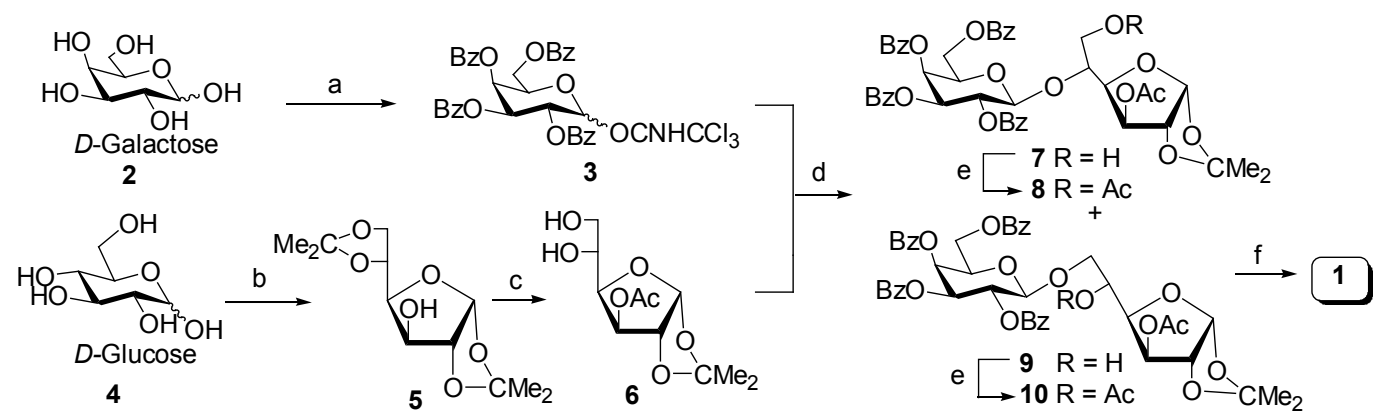

Reagents and conditions: (a) (1) $\mathrm{BzCl}, \mathrm{Py}$; (2) $\mathrm{MeOH}-\mathrm{NH}_{3}$; (3) $\mathrm{CCl}_{3} \mathrm{CN}, \mathrm{K}_{2} \mathrm{CO}_{3}, 84 \%$ for three steps; (b) Acetone, $\mathrm{H}_{2} \mathrm{SO}_{4}, 81 \%$; (c) (1) $\mathrm{Ac} 2 \mathrm{O}$,

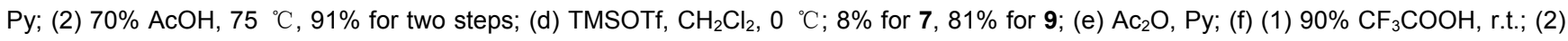
$\mathrm{MeONa}$, r.t.

\section{Scheme 2}

(a)

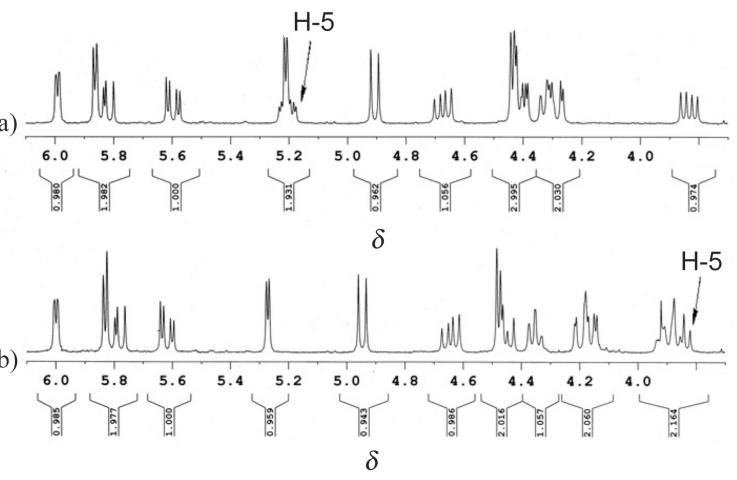

(c)

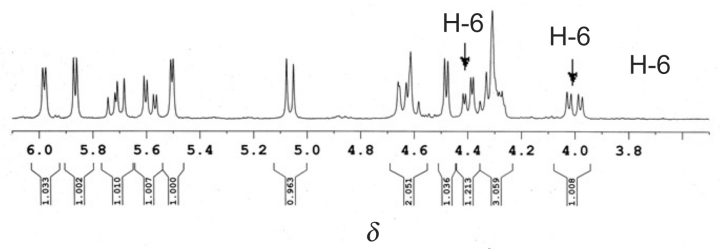

(d)

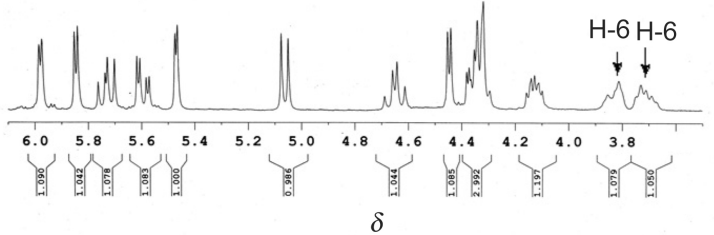

图 1 (a)化合物 10、(b)化合物 9、(c)化合物 8 和(d)化合物 7 的局部放大核磁图

Figure $1{ }^{1}$ H NMR partial enlarged spectrogram of compounds 10 (a), 9 (b), 8 (c) and 7 (d) 
线大量制备目标双糖的应用受到一定限制, 因此我们进 一步探索了异乳糖的逐步合成法.

之前在多个生物活性分子的合成中, 课题组探索了 酰基对羟基的选择性保护方法 ${ }^{[10,14,15]}$, 重点发展了烯丙 氧羰基作为鿒糖合成的选择性正交保护基的脱除方 法 $^{[16]}$, 并应用该方法完成了多个糖环羟基的选择性保护 以及多个复杂的重要生物活性的寡糖的首次合成 ${ }^{[17 \sim 20]}$. 该保护基在缓冲剂乙酸铵存在、四三苯基膦钯催化下, 可以在 $5 \mathrm{~min}$ 内以嗍氢化钠近似定量脱除而不影响分子 中其他众多基团, 尤其是酰基. 因此在异乳糖逐步合成 路线设计中, 我们优先选择了烯丙氧羰基作为正交保护 基团. 如 Scheme 3 所示, 化合物 6 先在烯丙氧羰基氯、 吡啶作用下选择性在 6 位引入烯丙氧羰基保护基，得到 化合物 11, 收率 $97 \%$. 其后在苯甲酰氯及吡定条件下在 5 位苯甲酰基化, 定量得到 1,2- $O$-异丙叉-3- $O$-乙酰 基-5- $O$ - 苯甲酰基- 6 - $O$-烯丙氧羰基- $\alpha$ - $D$-呋喃葡萄糖(12). 化合物 13 在四三苯基膦钯、硼氢化钠作用下脱除 6 位 烯丙氧羰基 ${ }^{[16 ~ 19]}$, 即得到糖基受体 1,2- $O$-异丙叉-3- $O$ 乙酰基-5- $O$-苯甲酰基- $\alpha$ - $D$-呋喃葡萄糖(13)，收率 $95 \%$. 酰化反应的区域选择性可以方便地通过对比化合物 11, 12 和 13 氢谱的化学位移变化确证(表 1).

表 1 酰化反应区域选择性特征峰化学位移

Table 1 Feature chemical shift of regioselective acylation reactions

\begin{tabular}{clll}
\hline$\delta$ & \multicolumn{1}{c}{$\mathbf{1 1}$} & \multicolumn{1}{c}{$\mathbf{1 2}$} & \multicolumn{1}{c}{$\mathbf{1 3}$} \\
\hline$\delta_{\mathrm{H}-5}$ & $3.90(1 \mathrm{H})$ & $5.49(1 \mathrm{H})$ & $5.35(1 \mathrm{H})$ \\
$\delta_{\mathrm{H}-6}$ & $4.18 \sim 4.28(2 \mathrm{H})$ & $4.65 \sim 4.71(2 \mathrm{H})$ & $3.95 \sim 4.07(2 \mathrm{H})$ \\
\hline
\end{tabular}

研究发现, 化合物 13 的合成可以通过三步连续操 作获得, 中途无需经柱纯化, 从而大大简化合成步骤, 使目标化合物的批量制备成为可能. 即 $1,2-O$ - 异丙 叉-3- $O$-乙酰基- $\alpha$ - $D$-呋喃葡萄糖(6)的 6 位稀丙氧糖基 化、5 位苯甲酰化可经一锅法反应得到, 在经简单后处 理得到粗产品 12 后不分离纯化，直接在四三苯基膦钯、
硼氢化钠条件下脱除 6 位烯丙氧羰基，经过很短的硅胶 柱纯化, 即可以 $88 \%$ 的收率得到纯品单糖受体 13. 单糖 受体 13 和单糖供体 3 在 TMSOTf 的催化下进行偶联反 应，得到 $\beta-1 \rightarrow 6$ 连接的双糖 14. 化合物 14 的糖苷键的 $\beta$ 构型通过 $\delta 4.98$ 处的 H-1 和 H-2 之间的偶合常数 $\left(J_{1,2}=\right.$ 8.0)得到确认. 随后，化合物 $\mathbf{1 4}$ 在 $90 \%$ 三氟乙酸作用下 水解脱除 1,2-O-异丙叉基，所得产物在甲醇钠作用下脱 除全部保护基团以 90\%的产率得到化合物 1. 化合物 1 的结构经 ${ }^{13} \mathrm{C} \mathrm{NMR}$ 确证即为异乳糖, 所合成二糖结构由 $\delta 105.38\left(\mathrm{C}-1^{\prime}\right), 98.07(\mathrm{C}-1 \beta), 94.23(\mathrm{C}-1 \alpha)$ 处的 3 个异头 碳的信号峰得以确证，此外，还可以观察到半乳糖环 4 位碳的信号峰 $(\delta 71.61)$. 所有的数据均与文献数据吻 合 ${ }^{[21]}$.

\section{2 结论}

本文以 $D$-葡萄糖、 $D$-半乳糖为起始原料, 探索了异 乳糖的多羟基区域选择性偶联与非选择性偶联两条合 成路线，其中非选择性偶联路线以烯丙氧羰基为正交保 护基，经过选择性保护、脱保护、糖基化等五锅反应，实 现了异乳糖的合成，总收率达 $44 \%$. 其中多个化合物经 多步一锅反应得到，不仅缩短了反应步骤，还能以较高 的产率得到目标中间体，使该化合物的十克级别批量制 备成为可能. 该合成路线对于获取异乳糖及其衍生物, 进行生物活性研究具有重要的意义.

\section{3 实验部分}

\section{1 仪器与试剂}

质谱采用 Bruker Daltonics Bio-TOF-Q III 型质谱 仪(ESIMS)测定; 核磁共振波谱使用 Bruker Avance 600 型核磁共振仪测定(四甲基硅烷 TMS 为内标). 所 有试剂为国产市售分析纯，无水溶剂按标准方法处 理.

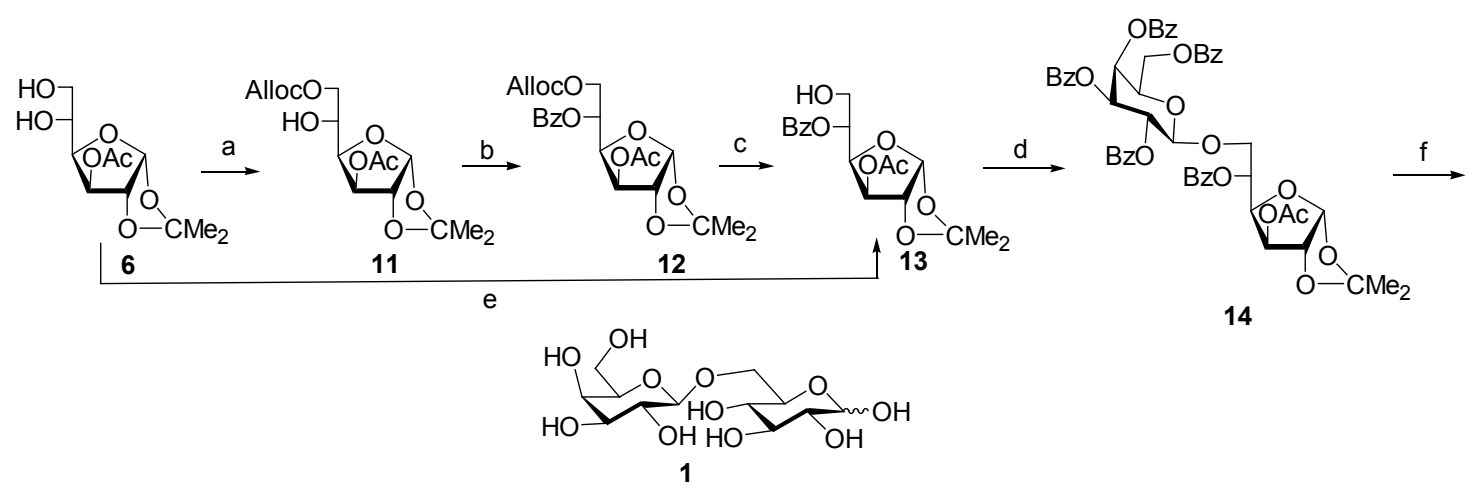

Reagents and conditions: (a) AllocCl, $\mathrm{Py}, \mathrm{CH}_{2} \mathrm{Cl}_{2}, 97 \%$; (b) $\mathrm{BzCl}, \mathrm{Py}, 100 \%$; (c) $\mathrm{Pd}_{2}\left[\mathrm{PPh}_{3}\right]_{4}, \mathrm{NaBH}_{4}, \mathrm{CH}_{3} \mathrm{COONH}_{4}, \mathrm{CH}_{3} \mathrm{OH}-\mathrm{THF}, 0{ }^{\circ} \mathrm{C}, 95 \%$; (d) 3, TMSOTf, $\mathrm{CH}_{2} \mathrm{Cl}_{2}, 0{ }^{\circ} \mathrm{C}, 80 \%$; (e) a, b, c, $88 \%$ for three steps; (f) $90 \% \mathrm{CF}_{3} \mathrm{COOH}$, r.t.; $\mathrm{CH}_{3} \mathrm{ONa}$, r.t., $90 \%$

\section{Scheme 3}




\section{2 实验方法}

3.2.12,3,4,6-四- $O$ - 苯甲酰基- $D$-半乳糖三氯乙酰亚 胺酯(3)的制备

在装有机械搅拌、回流冷凝管的 $1 \mathrm{~L}$ 三口瓶中, 将 化合物 $2(18 \mathrm{~g}, 0.1 \mathrm{~mol})$ 悬浮在 $250 \mathrm{~mL}$ 干燥的甲苯中, 搅拌下加入 $\mathrm{Py}(48 \mathrm{~mL}, 0.6 \mathrm{~mol}), 30 \mathrm{~min}$ 内滴加 $\mathrm{BzCl}(64$ $\mathrm{mL}, 0.55 \mathrm{~mol}$ )的 $60 \mathrm{~mL}$ 甲苯溶液. 滴加完毕加热至回流, TLC $[V$ (石油醚 $): V($ 乙酸乙酯 $)=3: 1]$ 检测反应完全. 冷却到 $0{ }^{\circ} \mathrm{C}$, 过滤除去吡啶盐酸盐, 向滤液向体系中加 入 $400 \mathrm{~mL}$ 饱和甲醇氨溶液, 室温下搅拌反应, 及时监 测至反应结束. 过滤除去不溶物, 低温浓缩除去溶剂, 真 空干燥 $2 \mathrm{~h}$, 氮气保护下加入 $800 \mathrm{~mL}$ 无水 $\mathrm{CH}_{2} \mathrm{Cl}_{2}$, 加入 $\mathrm{CCl}_{3} \mathrm{CN} 15 \mathrm{~mL}(0.15 \mathrm{~mol}) 、 \mathrm{~K}_{2} \mathrm{CO}_{3} 69 \mathrm{~g}(0.5 \mathrm{mmol})$, 室温 反应过夜, TLC $[V$ (石油醚) $: V$ (乙酸乙酯 $)=3: 1]$ 检测反 应完全. 抽滤除去不溶物后浓缩, 柱层析纯化得到化合 物 $\mathbf{3}^{[22]}\left(62 \mathrm{~g}\right.$, 收率 84\%). $[\alpha]_{\mathrm{D}}^{25}+120\left(c 0.014, \mathrm{CHCl}_{3}\right)$. ${ }^{1} \mathrm{H}$ NMR $\left(\mathrm{CDCl}_{3}, 300 \mathrm{MHz}\right) \delta: 4.42\left(\mathrm{dd}, J_{5,6 \mathrm{a}}=7.0 \mathrm{~Hz}\right.$, $\left.J_{6 \mathrm{a}, 6 \mathrm{~b}}=11.4 \mathrm{~Hz}, 1 \mathrm{H}, \mathrm{H}-6 \mathrm{a}\right), 4.62\left(\mathrm{dd}, J_{5,6 \mathrm{~b}}=6.0 \mathrm{~Hz}, 1 \mathrm{H}\right.$, H-6b), $4.82 \sim 4.88(\mathrm{~m}, 1 \mathrm{H}, \mathrm{H}-5), 5.95\left(\mathrm{dd}, J_{1,2}=3.6 \mathrm{~Hz}\right.$, $\left.J_{2,3}=10.6 \mathrm{~Hz}, 1 \mathrm{H}, \mathrm{H}-2\right), 6.06$ (dd, $J_{2,3}=10.6 \mathrm{~Hz}, J_{3,4}=3.3$ $\mathrm{Hz}, 1 \mathrm{H}, \mathrm{H}-3), 6.16\left(\mathrm{~d}, J_{3,4}=3.3 \mathrm{~Hz}, 1 \mathrm{H}, \mathrm{H}-4\right), 6.91$ (d, $\left.J_{1,2}=3.6 \mathrm{~Hz}, 1 \mathrm{H}, \mathrm{H}-1\right), 7.29 \sim 7.54(\mathrm{~m}, 13 \mathrm{H}, \mathrm{Ar}-\mathrm{H}), 7.78 \sim$ $8.10(\mathrm{~m}, 7 \mathrm{H}, \mathrm{Ar}-\mathrm{H}), 8.63\left(\mathrm{~s}, 1 \mathrm{H}, \mathrm{OCNHCCl}_{3}\right)$

$3.2 .21,2: 5,6-O$ - 双异丙叉- $\alpha-D$-呋喃葡萄糖(5)的制备

在 $1000 \mathrm{~mL}$ 三口瓶中加入丙酮 $640 \mathrm{~mL}$ 、无水葡萄 糖 $30 \mathrm{~g}$, 并逐滴加入浓 $\mathrm{H}_{2} \mathrm{SO}_{4} 24 \mathrm{~mL}$, 控制反应温度在 $25{ }^{\circ} \mathrm{C}$ 以下, 滴加完毕后维持反应温度 $20{ }^{\circ} \mathrm{C}$ 左右, 反应 $3 \mathrm{~h}$, TLC 检测 $[V$ (石油醚) $: V($ 乙酸乙酯 $)=3: 1$ ]反应进 程直到反应完全.

向反应体系中加入 $20 \% \mathrm{NaOH}$ 溶液进行中和反应, 控制温度在 $25{ }^{\circ} \mathrm{C}$ 以下. 中和完毕过滤, 滤出固体用少 量丙酮洗涤, 洗涤液与前滤液合并, 二氯甲烷萃取, 无 水 $\mathrm{Na}_{2} \mathrm{SO}_{4}$ 干燥, 浓缩, 用 $V$ (石油醚) $: V($ 乙酸乙酯 $)=$ 5: 1 重结晶得白色固体产物 $35 \mathrm{~g}$, 收率 $81 \%$. 熔点 $104 \sim 108{ }^{\circ} \mathrm{C},[\alpha]_{\mathrm{D}}^{25}-11.4\left(c \quad 0.033, \mathrm{CHCl}_{3}\right)$ [文献值 ${ }^{[23]}$ 熔点 $\left.105 \sim 108{ }^{\circ} \mathrm{C},[\alpha]_{\mathrm{D}}^{25}-12.5\left(c 0.033, \mathrm{CHCl}_{3}\right)\right]$.

3.2.3 1,2- $O$-异丙叉-3- $O$-乙酰基- $\alpha$ - $D$ - 呋喃葡萄糖(6) 的制备

于 $250 \mathrm{~mL}$ 圆底烧瓶中, 将 1,2:5,6-O-双异丙叉基$\alpha$-D -呋喃葡萄糖(5) $26 \mathrm{~g}(0.10 \mathrm{~mol})$ 加入到 Py $50 \mathrm{~mL}$ 、 $\mathrm{Ac}_{2} \mathrm{O} 19 \mathrm{~mL}(0.20 \mathrm{mmol})$ 的混合液中, 置室温下搅拌反 应过夜. TLC 检测 $[V$ (石油醚) $: V($ 乙酸乙酯 $)=2: 1$ ]反应 完全. 减压旋蒸掉溶剂, 用 $20 \mathrm{~mL} \times 2$ 甲苯共蒸除尽 $\mathrm{Ac}_{2} \mathrm{O}$ 和 $\mathrm{Py}$, 得 1,2:5,6-O-双异丙叉-3- $O$-乙酰基- $\alpha$ - $D$-呋 喃葡萄糖的粗品. 所得粗产品溶于 $200 \mathrm{~mL} 70 \% \mathrm{HOAc}$,
$75{ }^{\circ} \mathrm{C}$ 下反应 $1.5 \mathrm{~h}, \mathrm{TLC}$ 检测 $[V$ (石油梄)：V(乙酸乙 酯 $)=1: 1$ 反 反应完全. 减压旋蒸掉溶剂, 浓缩物用 $(20$ $\mathrm{mL} \times 2$ ) 甲苯共蒸, 得到 1,2- $O$-异丙叉-3-O-乙酰基- $\alpha$ - $D$ 呋喃葡萄糖的粗品. 该粗品用石油醚、乙酸乙酯重结晶 得到白色粉末状纯品化合物 6 (23.8 g, 收率 97\%). 熔点 $124 \sim 128{ }^{\circ} \mathrm{C},[\alpha]_{\mathrm{D}}^{25}+20\left(c 0.038, \mathrm{CHCl}_{3}\right)$ [文献值 ${ }^{[24]}$ : 熔 点 $\left.126 \sim 126.5{ }^{\circ} \mathrm{C},[\alpha]_{\mathrm{D}}^{22}+18\left(c 0.93, \mathrm{CHCl}_{3}\right)\right]$.

$3.2 .42,3,4,6$ - 四- $O$ - 苯甲酰基- $\beta$ - $D$ - 半乳糖 $-(1 \rightarrow 5)$ 1,2- $O$ - 异丙叉- $3-O$ - 乙酰基- $\alpha$ - $D$ - 呋喃葡萄糖 (7) 和 $2,3,4,6$ - 四- $O$ - 苯甲酰基- $\beta$ - $D$-半乳糖- $(1 \rightarrow 6)-1,2-O$ - 异丙 叉-3- $O$-乙酰基- $\alpha$ - D-呋喃葡萄糖(9)的制备

将化合物 6 (0.26 g, $1 \mathrm{mmol}) 、$ 化合物 $3(0.81 \mathrm{~g}, 1.1$ $\mathrm{mmol}$ )和 $4 \AA$ 分子篎 $(1 \mathrm{~g}$ )溶于 $20 \mathrm{~mL}$ 干燥二氯甲烷中, 冰浴冷却至 $-10{ }^{\circ} \mathrm{C}$, 氮气保护下搅拌 $0.5 \mathrm{~h}$, 加入三甲 基硅烷基三氟甲磺酸酯(TMSOTf) $8 \mu \mathrm{L}$ ，保持 $-10{ }^{\circ} \mathrm{C}$ 反 应 $0.5 \mathrm{~h}$, 撤去冰浴使反应物自然升温到室温, $2 \mathrm{~h}$ 后 TLC $[V$ (石油醚) $: V$ (乙酸乙酯 $)=2: 1]$ 检测反应完全, 加 $10 \mu \mathrm{L}$ 三乙胺终止反应. 抽滤反应液除去 $4 \AA$ 分子篮, 滤液减压下浓缩, 柱层析分离 $[V$ (石油醚) : $V$ ( 乙酸乙 酯) $=6:$ 1]得白色固体双糖 $7(67 \mathrm{mg}, 8 \%)$ 和 $9(680 \mathrm{mg}$, $81 \%)$.

化合物 7: $[\alpha]_{\mathrm{D}}^{25}+66\left(c \quad 0.012, \mathrm{CHCl}_{3}\right) ;{ }^{1} \mathrm{H}$ NMR $\left(\mathrm{CDCl}_{3}, 300 \mathrm{MHz}\right) \delta: 1.28,1.49\left(2 \mathrm{~s}, 6 \mathrm{H}, \mathrm{CMe}_{2}\right), 2.15(\mathrm{~s}$, $\left.3 \mathrm{H}, \mathrm{COCH}_{3}\right), 2.87(\mathrm{~d}, J=19.7 \mathrm{~Hz}, 1 \mathrm{H}, \mathrm{OH}), 3.71 \sim 3.90$ (m, 2H, H-6a', H-6b'), 4.12 (m, 1H, H-5'), 4.29 4.38 (m, $3 \mathrm{H}, \mathrm{H}-5, \mathrm{H}-6 \mathrm{a}, \mathrm{H}-6 \mathrm{~b}), 4.44$ (d, $\left.J_{1^{\prime}, 2^{\prime}}=3.6 \mathrm{~Hz}, 1 \mathrm{H}, \mathrm{H}-2^{\prime}\right)$, $4.61 \sim 4.65$ (m, 1H, H-4'), 5.05 (d, $\left.J_{1,2}=7.8 \mathrm{~Hz}, 1 \mathrm{H}, \mathrm{H}-1\right)$, $5.46\left(\mathrm{~d}, J_{2^{\prime}, 3^{\prime}}=2.6 \mathrm{~Hz}, 1 \mathrm{H}, \mathrm{H}-3^{\prime}\right), 5.59\left(\mathrm{dd}, J_{2,3}=10.4 \mathrm{~Hz}\right.$, $\left.J_{3,4}=3.3 \mathrm{~Hz}, 1 \mathrm{H}, \mathrm{H}-3\right), 5.72\left(\mathrm{dd}, J_{1,2}=7.8 \mathrm{~Hz}, J_{2,3}=10.4\right.$ $\mathrm{Hz}, 1 \mathrm{H}, \mathrm{H}-2), 5.83\left(\mathrm{~d}, J_{1^{\prime}, 2^{\prime}}=3.6 \mathrm{~Hz}, 1 \mathrm{H}, \mathrm{H}-1^{\prime}\right), 5.97$ (dd, $\left.J_{3,4}=J_{4,5}=3.3 \mathrm{~Hz}, 1 \mathrm{H}, \mathrm{H}-4\right), 7.21 \sim 7.26(\mathrm{~m}, 2 \mathrm{H}, \mathrm{Ar}-\mathrm{H})$, $7.37 \sim 7.63$ (m, 10H, Ar-H), 7.75 8.11 (m, 8H, Ar-H); ${ }^{13} \mathrm{C} \mathrm{NMR}\left(75 \mathrm{MHz}, \mathrm{CDCl}_{3}\right) \delta: 169.38,165.96,165.44$, $165.26,233.55,133.40,133.22,129.99,129.73,129.69$, $129.58,129.28,129.01,128.94,128.65,128.62,128.47$, $128.38,128.21,112.35,104.68,100.51,82.86,78.32$, $76.29,75.56,71.60,71.23,70.20,67.79,26.60,26.08$, 20.89. HRMS (ESI) calcd for $\mathrm{C}_{45} \mathrm{H}_{45} \mathrm{O}_{16}(\mathrm{M}+\mathrm{H})^{+}$ 841.27021, found 841.26678.

化合物 9: $[\alpha]_{\mathrm{D}}^{25}+78\left(c \quad 0.012, \mathrm{CHCl}_{3}\right) ;{ }^{1} \mathrm{H}$ NMR $\left(\mathrm{CDCl}_{3}, 300 \mathrm{MHz}\right) \delta: 1.27,1.41\left(2 \mathrm{~s}, 6 \mathrm{H}, \mathrm{CMe}_{2}\right), 2.07(\mathrm{~s}$, $3 \mathrm{H}, \mathrm{COCH}_{3}$ ), 2.77 (br s, $\left.1 \mathrm{H}, \mathrm{OH}\right), 3.82 \sim 3.93(\mathrm{~m}, 2 \mathrm{H}$, H-5', H-6a), 4.13 4.21 (m, 2H, H-6a', H-6b'), 4.32 4.37 (m, 1H, H-5), 4.42 4.48 (m, 2H, H-2', H-4'), 4.65 (dd, $\left.J_{5,6 \mathrm{~b}}=6.5 \mathrm{~Hz}, J_{6 \mathrm{a}, 6 \mathrm{~b}}=11.2 \mathrm{~Hz}, 1 \mathrm{H}, \mathrm{H}-6 \mathrm{~b}\right), 4.93$ (d, $J_{1,2}=7.9$ 
$\mathrm{Hz}, 1 \mathrm{H}, \mathrm{H}-1), 5.26\left(\mathrm{~d}, J_{2^{\prime}, 3^{\prime}}=2.8 \mathrm{~Hz}, 1 \mathrm{H}, \mathrm{H}-3^{\prime}\right), 5.60$ (dd, $\left.J_{2,3}=10.4 \mathrm{~Hz}, J_{3,4}=3.4 \mathrm{~Hz}, 1 \mathrm{H}, \mathrm{H}-3\right), 5.76 \sim 5.83(\mathrm{~m}, 2 \mathrm{H}$, H-1', H-2), 5.99 (dd, $\left.J_{3,4}=J_{4,5}=3.4 \mathrm{~Hz}, 1 \mathrm{H}, \mathrm{H}-4\right), 7.23 \sim$ $7.26(\mathrm{~m}, 2 \mathrm{H}, \mathrm{Ar}-\mathrm{H}), 7.39 \sim 7.62(\mathrm{~m}, 10 \mathrm{H}, \mathrm{Ar}-\mathrm{H}), 7.75 \sim$ 7.78 (m, 2H, Ar-H), $7.93 \sim 7.95$ (m, 2H, Ar-H), $7.95 \sim 8.09$ $(\mathrm{m}, 4 \mathrm{H}, \mathrm{Ar}-\mathrm{H}) ;{ }^{13} \mathrm{C}$ NMR $\left(75 \mathrm{MHz}, \mathrm{CDCl}_{3}\right) \delta: 165.91$, $165.41,165.38,165.36,133.49,133.21,133.17,129.88$, $129.71,129.63,129.26,129.08,128.91,128.63,128.52$, $128.34,128.16,112.15,104.82,101.92,82.98,78.47$, $77.42,77.00,76.57,75.89,72.01,71.52,71.43,69.90$, $68.05,67.44,61.93,26.50,26.10,20.60$. HRMS (ESI) calcd for $\mathrm{C}_{45} \mathrm{H}_{48} \mathrm{NO}_{16}\left(\mathrm{M}+\mathrm{NH}_{4}\right)^{+}$858.29474, found 858.29676 .

3.2.5 2,3,4,6-四- $O$ - 苯甲酰基- $\beta$ - $D$ - 半乳糖- $(1 \rightarrow 5)$ 1,2- $O$ - 异丙叉- 3,6 -二- $O$-乙酰基- $\alpha$ - $D$ - 呋喃葡萄糖 $(8)$ 和 $2,3,4,6$-四- $O$ - 苯甲酰基- $\beta$ - $D$-半乳糖-( $1 \rightarrow 6$ )- $1,2-O$ - 异丙 叉- 3,5 -二- $O$ - 乙酰基- $\alpha-D$ - 呋喃葡萄糖(10)的制备

将化合物 7 (50 mg, $0.06 \mathrm{mmol}$ )溶于 $5 \mathrm{~mL}$ 干燥吡啶 中, 加入乙酸酎 $3 \mathrm{~mL}$, 室温下反应 $2 \mathrm{~h}$, TLC [ $V$ (石油 醚) $: V($ 乙酸乙酯 $)=2: 1$ 检测反应完全, 直接浓缩, 甲 苯共蒸 $(10 \mathrm{~mL} \times 3)$ 除去溶剂后柱层析分离 $[V$ (石油醚) : $V($ 乙酸乙酯 $)=5 ： 1$ ]得白色粉末状固体 $8(50 \mathrm{mg}, 96 \%)$. 化合物 10 按上述方法制备.

化合物 8: $[\alpha]_{\mathrm{D}}^{25}+72\left(c 0.011, \mathrm{CHCl}_{3}\right) ;{ }^{1} \mathrm{H}$ NMR $\left(\mathrm{CDCl}_{3}, 300 \mathrm{MHz}\right) \delta: 1.30,1.52\left(2 \mathrm{~s}, 6 \mathrm{H}, \mathrm{CMe}_{2}\right), 1.59(\mathrm{~s}$, $\left.3 \mathrm{H}, \mathrm{COCH}_{3}\right), 2.15\left(\mathrm{~s}, 3 \mathrm{H}, \mathrm{COCH}_{3}\right), 3.98\left(\mathrm{dd}, J_{5,6 \mathrm{a}}=4.5\right.$ $\left.\mathrm{Hz}, J_{6 \mathrm{a}^{\prime}, 6 \mathrm{~b}^{\prime}}=12.7 \mathrm{~Hz}, 1 \mathrm{H}, \mathrm{H}-6 \mathrm{a}^{\prime}\right), 4.27 \sim 4.35$ (m, 3H, H-5', H-6a, H-6b), 4.40 (d, $\left.J_{1^{\prime}, 2^{\prime}}=3.6 \mathrm{~Hz}, 1 \mathrm{H}, \mathrm{H}-2^{\prime}\right), 4.61 \sim 4.65$ (m, 2H, H-4', H-5), 5.05 (d, $J_{1,2}=7.7 \mathrm{~Hz}, 1 \mathrm{H}, \mathrm{H}-1$ ), 5.50 (d, $\left.J_{2^{\prime}, 3^{\prime}}=2.7 \mathrm{~Hz}, 1 \mathrm{H}, \mathrm{H}-3^{\prime}\right), 5.59$ (dd, $J_{2,3}=10.4 \mathrm{~Hz}, J_{3,4}=$ $3.3 \mathrm{~Hz}, 1 \mathrm{H}, \mathrm{H}-3), 5.72\left(\mathrm{dd}, J_{1,2}=7.7 \mathrm{~Hz}, J_{2,3}=10.4 \mathrm{~Hz}\right.$, $1 \mathrm{H}, \mathrm{H}-2), 5.83\left(\mathrm{~d}, J_{1^{\prime}, 2^{\prime}}=3.6 \mathrm{~Hz}, 1 \mathrm{H}, \mathrm{H}-1^{\prime}\right), 5.97$ (dd, $J_{3,4}=$ $\left.J_{4,5}=3.3 \mathrm{~Hz}, 1 \mathrm{H}, \mathrm{H}-4\right), 7.23 \sim 7.26(\mathrm{~m}, 2 \mathrm{H}, \mathrm{Ar}-H), 7.39 \sim$ 7.56 (m, 10H, Ar-H), $7.75 \sim 8.13(\mathrm{~m}, 8 \mathrm{H}, \mathrm{Ar}-\mathrm{H}) ;{ }^{13} \mathrm{C} \mathrm{NMR}$ $\left(75 \mathrm{MHz}, \mathrm{CDCl}_{3}\right) \delta: 170.38,169.21,165.97,165.48$, $165.44,164.81,166.59,133.34,133.21,130.08,129.72$, $129.66,129.62,129.33,129.21,128.91,128.68,128.47$, $128.35,128.30,128.22,112.43,104.83,99.03,83.08$, $75.09,72.34,71.68,71.28,69.81,67.74,62.49,61.70$, 26.71, 26.22, 20.92, 20.08. HRMS (ESI) calcd for $\mathrm{C}_{47} \mathrm{H}_{50} \mathrm{NO}_{17}\left(\mathrm{M}+\mathrm{NH}_{4}\right)^{+}$900.30591, found 900.30733 .

化合物 10: $[\alpha]_{\mathrm{D}}^{25}+20\left(c 0.011, \mathrm{CHCl}_{3}\right) ;{ }^{1} \mathrm{H}$ NMR $\left(\mathrm{CDCl}_{3}, 300 \mathrm{MHz}\right) \delta: 1.28,1.49\left(2 \mathrm{~s}, 6 \mathrm{H}, \mathrm{CMe}_{2}\right), 1.59(\mathrm{~s}$, $\left.3 \mathrm{H}, \mathrm{COCH}_{3}\right), 2.02\left(\mathrm{~s}, 3 \mathrm{H}, \mathrm{COCH}_{3}\right), 3.80\left(\mathrm{dd}, J_{5,6 \mathrm{a}}=5.7 \mathrm{~Hz}\right.$, $\left.J_{6 \mathrm{a}, 6 \mathrm{~b}}=11.2 \mathrm{~Hz}, 1 \mathrm{H}, \mathrm{H}-6 \mathrm{a}\right), 4.26 \sim 4.34(\mathrm{~m}, 2 \mathrm{H}, \mathrm{H}-6 \mathrm{a}$,
H-6b'), $4.38 \sim 4.44$ (m, 3H, H-2', H-4', H-5), 4.64 (dd, $\left.J_{5,6 \mathrm{~b}}=6.2 \mathrm{~Hz}, J_{6 \mathrm{a}, 6 \mathrm{~b}}=11.2 \mathrm{~Hz}, 1 \mathrm{H}, \mathrm{H}-6 \mathrm{~b}\right), 4.91\left(\mathrm{~d}, J_{1,2}=7.9\right.$ $\mathrm{Hz}, 1 \mathrm{H}, \mathrm{H}-1), 5.17 \sim 5.23$ (m, 2H, H-3', H-5'), 5.58 (dd, $\left.J_{2,3}=10.4 \mathrm{~Hz}, J_{3,4}=3.4 \mathrm{~Hz}, 1 \mathrm{H}, \mathrm{H}-3\right), 5.76 \sim 5.83(\mathrm{~m}, 2 \mathrm{H}$, H-1', H-2), 5.99 (dd, $\left.J_{3,4}=J_{4,5}=3.4 \mathrm{~Hz}, 1 \mathrm{H}, \mathrm{H}-4\right), 7.20 \sim$ $7.23(\mathrm{~m}, 2 \mathrm{H}, \mathrm{Ar}-\mathrm{H}), 7.39 \sim 7.61(\mathrm{~m}, 10 \mathrm{H}, \mathrm{Ar}-\mathrm{H}), 7.75 \sim$ 7.78 (m, 2H, Ar-H), $7.95 \sim 8.09$ (m, 6H, Ar-H); ${ }^{13} \mathrm{C}$ NMR $\left(75 \mathrm{MHz}, \mathrm{CDCl}_{3}\right) \delta: 169.47,169.29,165.91,165.43$, $165.41,164.95,133.45,133.14,129.90,129.72,129.68$, $129.66,129.34,129.26,128.97,128.67,128.51,128.36$, $128.24,128.16,112.18,104.96,101.33,83.16,76.61$, $74.54,71.49,71.30,69.63,68.47,68.25,68.03,61.74$, 26.61, 26.01, 20.59, 20.17. HRMS (ESI) calcd for $\mathrm{C}_{47} \mathrm{H}_{50} \mathrm{NO}_{17}\left(\mathrm{M}+\mathrm{NH}_{4}\right)^{+}$900.30542, found 900.30732 .

3.2.6 1,2- $O$-异丙叉-3- $O$-乙酰基-6- $O$-烯丙氧羰基- $\alpha$ $D$-呋喃葡萄糖 $(\mathbf{1 1})$ 的制备

将化合物 6 (7.86 g, $30.0 \mathrm{mmol}$ ) 溶解在 $100 \mathrm{~mL}$ 干燥 的 $\mathrm{CH}_{2} \mathrm{Cl}_{2}$ 中, 加入 $\mathrm{Py}(12 \mathrm{~mL}, 150.0 \mathrm{mmol})$, 冰浴冷却至 $-10{ }^{\circ} \mathrm{C}, 30 \mathrm{~min}$ 内滴加 AllocCl (3.39 mL, $\left.31.5 \mathrm{mmol}\right)$ 的 $40 \mathrm{mLCH}_{2} \mathrm{Cl}_{2}$ 溶液. 滴加完毕撤去冰浴, 室温摚拌 $3 \mathrm{~h}$, TLC $[V$ (石油醚) $: V($ 乙酸乙酯 $)=3: 1$ ]检测反应完全. 反应体系依次用 $1 \mathrm{~mol} / \mathrm{L}$ 盐酸和饱和 $\mathrm{NaHCO}_{3}$ 溶液、饱 和食盐水洗涤, $\mathrm{CH}_{2} \mathrm{Cl}_{2}$ 萃取、无水 $\mathrm{Na}_{2} \mathrm{SO}_{4}$ 干燥、浓缩, 柱 层析 $[V($ 石油醚 $) ： V($ 乙酸乙酯 $)=4: 1]$ 分离得浅黄色粘 稠状液体 11 (10 g, 收率 97\%). [ $\alpha]_{\mathrm{D}}^{25}+108$ (c 0.029 , $\left.\mathrm{CHCl}_{3}\right) ;{ }^{1} \mathrm{H} \mathrm{NMR}\left(\mathrm{CDCl}_{3}, 300 \mathrm{MHz}\right) \delta: 1.31,1.51(2 \mathrm{~s}, 6 \mathrm{H}$, $\mathrm{CMe}_{2}$ ), 2.13 (s, 3H, $\left.\mathrm{COCH}_{3}\right), 2.94$ (d, $J=4.5 \mathrm{~Hz}, 1 \mathrm{H}, \mathrm{OH}$ ), $3.88 \sim 3.92(\mathrm{~m}, 1 \mathrm{H}, \mathrm{H}-5), 4.18 \sim 4.28(\mathrm{~m}, 2 \mathrm{H}, \mathrm{H}-6), 4.45$ $\left(\mathrm{dd}, J_{3,4}=2.4 \mathrm{~Hz}, J_{4,5}=11.4 \mathrm{~Hz}, 1 \mathrm{H}, \mathrm{H}-4\right), 4.54$ (d, $J_{1,2}=$ $3.6 \mathrm{~Hz}, 1 \mathrm{H}, \mathrm{H}-2), 4.62,4.64\left(2 \mathrm{t}, J=1.3 \mathrm{~Hz}, 2 \mathrm{H}, \mathrm{CH}_{2} \mathrm{CH}-\right.$ $\left.\mathrm{CH}_{2} \mathrm{O}\right), 5.24 \sim 5.40\left(\mathrm{~m}, 3 \mathrm{H}, \mathrm{H}-3, \mathrm{CH}_{2} \mathrm{CHCH}_{2}\right), 5.86 \sim 5.99$ (m, 2H, H-1, $\mathrm{CH}_{2} \mathrm{CHCH}_{2}$ ); ${ }^{13} \mathrm{C}$ NMR (75 MHz, $\mathrm{CDCl}_{3}$ ) $\delta$ : $170.48,154.89,131.30,118.76,112.21,104.76,82.85$, 78.43, 76.08, 69.40, 68.46, 66.59, 26.46, 26.04, 20.58. HRMS (ESI) calcd for $\mathrm{C}_{15} \mathrm{H}_{23} \mathrm{O}_{9}(\mathrm{M}+\mathrm{H})^{+} 347.13366$, found 347.13339 .

3.2.7 1,2- $O$-异丙叉-3- $O$-乙酰基-5- $O$ - 苯甲酰基-6- $O$ 烯丙氧羰基- $\alpha-D-$-呋喃葡萄糖(12)的制备

将化合物 10 (10.4 g, $30 \mathrm{mmol}$ )溶解于 $60 \mathrm{~mL}$ 干燥 吡啶中, 冰浴下滴加 $\mathrm{BzCl}(4.2 \mathrm{~mL}, 36 \mathrm{mmol})$, 滴加完毕 后冰浴下摚拌 $30 \mathrm{~min}$, 然后将体系移置室温下反应过 夜, TLC $[V$ (石油醚) $: V($ 乙酸乙酯 $)=3: 1$ ]检测反应完 全. 将反应体系用 $\mathrm{CH}_{2} \mathrm{Cl}_{2}$ 稀释，依次用 $1 \mathrm{~mol} / \mathrm{L}$ 盐酸、 饱和 $\mathrm{NaHCO}_{3}$ 溶液、饱和食盐水洗涤, $\mathrm{CH}_{2} \mathrm{Cl}_{2}$ 萃取、无 水 $\mathrm{Na}_{2} \mathrm{SO}_{4}$ 干燥、浓缩柱层析 $[V$ (石油醚) : $V$ (乙酸乙 
酯 $)=4: 1$ ]分离得浅黄色粘稠状液体 $12(13.5 \mathrm{~g}$, 收率 $100 \%) .[\alpha]_{\mathrm{D}}^{25}+120\left(c 0.022, \mathrm{CHCl}_{3}\right) ;{ }^{1} \mathrm{H}$ NMR $\left(\mathrm{CDCl}_{3}\right.$, $300 \mathrm{MHz}) \delta: 1.31,1.52\left(2 \mathrm{~s}, 6 \mathrm{H}, \mathrm{CMe}_{2}\right), 2.01(\mathrm{~s}, 3 \mathrm{H}$, $\left.\mathrm{COCH}_{3}\right), 4.40$ (dd, $\left.J=5.0 \mathrm{~Hz}, 12.2 \mathrm{~Hz}, 1 \mathrm{H}, \mathrm{H}-4\right), 4.51$ (d, $\left.J_{1,2}=3.6 \mathrm{~Hz}, 1 \mathrm{H}, \mathrm{H}-2\right), 4.57\left(\mathrm{t}, J=1.3 \mathrm{~Hz}, 1 \mathrm{H}, \mathrm{CH}_{2} \mathrm{CH}-\right.$ $\mathrm{CH}_{2} \mathrm{O}$ ), 4.59 (t, $J=1.3 \mathrm{~Hz}, 1 \mathrm{H}, \mathrm{CH}_{2} \mathrm{CHCH}_{2} \mathrm{O}$ ), $4.63 \sim 4.71$ (m, 2H, H-6), 5.18 $5.28\left(\mathrm{~m}, 2 \mathrm{H}, \mathrm{CH}_{2} \mathrm{CHCH}_{2} \mathrm{O}\right), 5.32 \sim$ $5.34(\mathrm{~m}, 1 \mathrm{H}, \mathrm{H}-3), 5.49 \sim 5.55$ (m, 1H, H-5), $5.80 \sim 5.93$ $\left(\mathrm{m}, 1 \mathrm{H}, \mathrm{CH}_{2} \mathrm{CHCH}_{2} \mathrm{O}\right), 5.96\left(\mathrm{~d}, \quad J_{1,2}=3.6 \mathrm{~Hz}, 1 \mathrm{H}, \mathrm{H}-1\right)$, $7.40 \sim 7.56$ (m, 3H, Ar-H), 7.96 8.00 (m, $2 \mathrm{H}, \mathrm{Ar}-\mathrm{H}) ;{ }^{13} \mathrm{C}$ NMR (75 MHz, $\left.\mathrm{CDCl}_{3}\right) \delta: 169.36,165.10,154.66,133.31$, $131.36,129.69,128.34,118.83,112.51,105.10,83.17$, $75.10,68.56,68.18,66.59,26.72,26.14,20.51$. HRMS (ESI) calcd for $\mathrm{C}_{22} \mathrm{H}_{27} \mathrm{O}_{10}(\mathrm{M}+\mathrm{H})^{+}$451.15987, found 451.15887 .

3.2.8 1,2- $O$-异丙叉-3- $O$-乙酰基-5- $O$ - 苯甲酰基- $\alpha$ - $D$ 呋喃葡萄糖(13)的制备

方法一: 于 $250 \mathrm{~mL}$ 圆底烧瓶中, 将化合物 $\mathbf{1 2}(9 \mathrm{~g}$, $20 \mathrm{mmol}$ )溶于 $200 \mathrm{~mL}[V$ (甲醇) $: V$ (四氢呋喃 $)=1 ： 1$ ] 溶 液中, 向体系中加入 $\mathrm{CH}_{3} \mathrm{COONH}_{4}(15.4 \mathrm{~g}, 200 \mathrm{mmol})$, 冰浴冷却至 $-10{ }^{\circ} \mathrm{C}$, 向体系中快速加入 $\mathrm{NaBH}_{4}(0.57 \mathrm{~g}$, $15 \mathrm{mmol}) 、 \mathrm{Pd}\left[\mathrm{PPh}_{3}\right]_{4}(0.92 \mathrm{~g}, 0.8 \mathrm{mmol}) 、 \mathrm{NaBH}_{4}(0.57 \mathrm{~g}$, $15 \mathrm{mmol})$. TLC [ $V$ (石油醚 $): V($ 乙酸乙酯 $)=2 ： 1]$ 检测至 反应完全. 将反应液减压浓缩, 除去甲醇、四氢呋喃. 将 反应体系用 $\mathrm{CH}_{2} \mathrm{Cl}_{2}$ 稀释，饱和食盐水萃取、无水 $\mathrm{Na}_{2} \mathrm{SO}_{4}$ 干燥、浓缩柱层析 $[V$ (石油醚) $: V$ (乙酸乙酯 $)=4: 1$ ]分离 得浅黄色粘稠状液体 13 (6.9 g, 收率 95\%). $[\alpha]_{\mathrm{D}}^{25}+88(c$ $\left.0.027, \mathrm{CHCl}_{3}\right) ;{ }^{1} \mathrm{H}$ NMR $\left(\mathrm{CDCl}_{3}, 300 \mathrm{MHz}\right) \delta: 1.32,1.55$ $\left(2 \mathrm{~s}, 6 \mathrm{H}, \mathrm{CMe}_{2}\right), 2.01\left(\mathrm{~s}, 3 \mathrm{H}, \mathrm{COCH}_{3}\right), 3.96\left(\mathrm{dd}, J_{5,6 \mathrm{a}}=4.3\right.$ $\left.\mathrm{Hz}, J_{6 \mathrm{a}, 6 \mathrm{~b}}=12.4 \mathrm{~Hz}, 1 \mathrm{H}, \mathrm{H}-6 \mathrm{a}\right), 4.07\left(\mathrm{dd}, J_{5,6 \mathrm{~b}}=3.0 \mathrm{~Hz}\right.$, $\left.J_{6 \mathrm{a}, 6 \mathrm{~b}}=12.4 \mathrm{~Hz}, 1 \mathrm{H}, \mathrm{H}-6 \mathrm{~b}\right), 4.52$ (d, $J_{1,2}=3.6 \mathrm{~Hz}, 1 \mathrm{H}, \mathrm{H}-2$ ), $4.67\left(\mathrm{dd}, J_{3,4}=3.0 \mathrm{~Hz}, J_{4,5}=9.3 \mathrm{~Hz}, 1 \mathrm{H}, \mathrm{H}-4\right), 5.31 \sim 5.37$ $(\mathrm{m}, 2 \mathrm{H}, \mathrm{H}-3, \mathrm{H}-5), 5.95\left(\mathrm{~d}, J_{1,2}=3.6 \mathrm{~Hz}, 1 \mathrm{H}, \mathrm{H}-1\right), 7.40 \sim$ 7.59 (m, 3H, Ar-H), $7.98 \sim 8.00$ (m, $2 \mathrm{H}, \mathrm{Ar}-\mathrm{H}) ;{ }^{13} \mathrm{C} \mathrm{NMR}$ $\left(75 \mathrm{MHz}, \mathrm{CDCl}_{3}\right) \delta: 169.37,165.57,133.26,131.36$, 129.60, 128.33, 112.39, 104.97, 83.11, 75.25, 71.09, 62.69, 26.60, 26.03, 20.46. HRMS (ESI) calcd for $\mathrm{C}_{18} \mathrm{H}_{23} \mathrm{O}_{8}(\mathrm{M}+$ $\mathrm{H})^{+} 367.13874$, found 367.13849 .

方法二(一锅法)：将化合物 6 (15.8 g, $60.0 \mathrm{mmol})$ 溶 解在 $100 \mathrm{~mL}$ 干燥的 $\mathrm{CH}_{2} \mathrm{Cl}_{2}$ 中, 加入 $\mathrm{Py}(24 \mathrm{~mL}, 0.3 \mathrm{~mol})$, 冰浴冷却至 $-10{ }^{\circ} \mathrm{C}, 30 \mathrm{~min}$ 内滴加 AllocCl $(6.8 \mathrm{~mL}, 63$ $\mathrm{mmol})$ 的 $60 \mathrm{mLCH}_{2} \mathrm{Cl}_{2}$ 溶液. 滴加完毕撤去冰浴, 室温 摚拌反应 $3 \mathrm{~h}$, TLC $[V$ (石油醚) $: V$ ( 乙酸乙酯 $)=3: 1$ ]检 测反应完全. 将反应液减压浓缩, 浓缩物用 $80 \mathrm{~mL}$ Py 溶 解, 加入 $\mathrm{BzCl}(8.4 \mathrm{~mL}, 72 \mathrm{mmol})$, 置室温下反应过夜,
TLC $[V$ (石油醚 $): V($ 乙酸乙酯 $)=4: 1]$ 检测反应完全. 将反应体系用 $\mathrm{CH}_{2} \mathrm{Cl}_{2}$ 稀释, 依次用 $1 \mathrm{~mol} / \mathrm{L}$ 盐酸、饱和 $\mathrm{NaHCO}_{3}$ 溶液、饱和食盐水洗涤, $\mathrm{CH}_{2} \mathrm{Cl}_{2}$ 萃取、无水 $\mathrm{Na}_{2} \mathrm{SO}_{4}$ 干燥, 浓缩后将剩余物溶于 $200 \mathrm{~mL}$ [ $V$ (甲醇) : $V($ 四氢 呋喃 $)=1:$ ] 溶液中, 向体系中加入 $\mathrm{CH}_{3} \mathrm{COONH}_{4}(46 \mathrm{~g}, 0.6 \mathrm{~mol})$, 冰浴冷却至 $-10{ }^{\circ} \mathrm{C}$, 向体 系中快速加入 $\mathrm{NaBH}_{4}(1.7 \mathrm{~g}, 45 \mathrm{mmol}) 、 \mathrm{Pd}\left[\mathrm{PPh}_{3}\right]_{4}(2.7 \mathrm{~g}$, $2.4 \mathrm{mmol}) 、 \mathrm{NaBH}_{4}(1.7 \mathrm{~g}, 45 \mathrm{mmol})$. TLC [ $V$ (石油醚) : $V($ 乙酸乙酯 $)=2: 1$ ]检测至反应完全. 将反应液减压浓 缩，除去甲醇、四氢呋喃. 将反应体系用 $\mathrm{CH}_{2} \mathrm{Cl}_{2}$ 稀释， 饱和食盐水萃取、无水 $\mathrm{Na}_{2} \mathrm{SO}_{4}$ 干燥、浓缩柱层析 $[V$ (石 油醚 $): V($ 乙酸乙酯 $)=6: 1$ ] 分离得浅黄色粘稠状液体 13 (6.4 g, 收率 $88 \%)$. 核磁数据与逐步合成法所得数据 吻合.

3.2.92,3,4,6-四- $O$ - 苯甲酰基- $\beta$ - $D$ - 半乳糖 $-(1 \rightarrow 6)$ 1,2- $O$ - 异丙叉-3- $O$-乙酰基-5- $O$ - 苯甲酰基- $\alpha$ - $D$ - 呋喃葡 萄糖(14)的制备

将化合物 $\mathbf{1 3}(11 \mathrm{~g}, 30 \mathrm{mmol}) 、$ 化合物 $3(24 \mathrm{~g}, 33$ $\mathrm{mmol})$ 和 $4 \AA$ 分子篎 $(8 \mathrm{~g})$ 溶于 $200 \mathrm{~mL}$ 干燥二氯甲烷中, 冰浴冷却至 $-10{ }^{\circ} \mathrm{C}$, 氮气保护下搅拌 $0.5 \mathrm{~h}$, 加入三甲 基硅烷基三氟甲磺酸酯(TMSOTf) $80 \mu \mathrm{L}$, 保持 $-10{ }^{\circ} \mathrm{C}$ 反应 $0.5 \mathrm{~h}$ ，撤去冰浴使反应物自然升温到室温, $3 \mathrm{~h}$ 后 TLC $[V$ (石油醚 $) ： V($ 乙酸乙酯 $)=2: 1]$ 检测反应完全, 加 $160 \mu \mathrm{L}$ 三乙胺结束反应. 抽滤反应液, 滤液减压下浓 缩, 柱层析分离 $[V$ (石油醚) $: V($ 乙酸乙酯 $)=6: 1]$ 得白 色固体双糖 $14(22.7 \mathrm{~g} \text {, 收率 80\%). [ } \alpha]_{\mathrm{D}}^{25}+114(c 0.01$, $\left.\mathrm{CHCl}_{3}\right) ;{ }^{1} \mathrm{H} \mathrm{NMR}\left(\mathrm{CDCl}_{3}, 300 \mathrm{MHz}\right) \delta: 1.28,1.54(2 \mathrm{~s}, 6 \mathrm{H}$, $\mathrm{CMe}_{2}$ ), 2.00 (s, 3H, $\mathrm{COCH}_{3}$ ), $4.08\left(\mathrm{dd}, J_{5^{\prime}, 6^{\prime}}=4.3 \mathrm{~Hz}\right.$, $\left.J_{6 a^{\prime}, 6 b^{\prime}}=12.0 \mathrm{~Hz}, 1 \mathrm{H}, \mathrm{H}-6 \mathrm{~b}^{\prime}\right), 4.31 \sim 4.39(\mathrm{~m}, 3 \mathrm{H}, \mathrm{H}-6 \mathrm{a}$, H-6b, H-6a'), 4.48 4.55 (m, 2H, H-2', H-5'), 4.74 (dd, $\left.J_{3,4}=3.0 \mathrm{~Hz}, J_{4,5}=9.3 \mathrm{~Hz}, 1 \mathrm{H}, \mathrm{H}-4^{\prime}\right), 5.00\left(\mathrm{~d}, J_{1,2}=8.0 \mathrm{~Hz}\right.$, $1 \mathrm{H}, \mathrm{H}-1), 5.23$ (d, $\left.J_{3,4}=3.0 \mathrm{~Hz}, 1 \mathrm{H}, \mathrm{H}-3^{\prime}\right), 5.40 \sim 5.45(\mathrm{~m}$, $1 \mathrm{H}, \mathrm{H}-5$ ), 5.58 (dd, $J_{2,3}=10.5 \mathrm{~Hz}, J_{3,4}=3.5 \mathrm{~Hz}, 1 \mathrm{H}, \mathrm{H}-3$ ), $5.84\left(\mathrm{dd}, J_{1,2}=8.0 \mathrm{~Hz}, J_{2,3}=10.5 \mathrm{~Hz}, 1 \mathrm{H}, \mathrm{H}-2\right), 5.90(\mathrm{~d}$, $\left.J=3.5 \mathrm{~Hz}, 1 \mathrm{H}, \mathrm{H}-1^{\prime}\right), 5.97\left(\mathrm{dd}, J_{3,4}=J_{4,5}=3.5 \mathrm{~Hz}, 1 \mathrm{H}\right.$, $\mathrm{H}-4), 7.18 \sim 8.07(\mathrm{~m}, 25 \mathrm{H}, \mathrm{Ar}-\mathrm{H}) ;{ }^{13} \mathrm{C}$ NMR $(75 \mathrm{MHz}$, $\left.\mathrm{CDCl}_{3}\right) \delta: 169.45,165.93,165.46,165.45,165.03,133.51$, $133.19,133.09,132.94,129.97,129.77,129.72,129.49$, $129.41,129.15,129.06,128.75,128.59,128.32,128.28$, $128.21,128.19,112.48,105.11,101.22,83.28,76.37$, $75.06,71.62,71.35,69.74,69.40,68.12,67.71,26.76$, 26.11, 20.58. HRMS (ESI) calcd for $\mathrm{C}_{52} \mathrm{H}_{52} \mathrm{NO}_{17}(\mathrm{M}+$ $\left.\mathrm{NH}_{4}\right)^{+}$962.32298, found 962.32025 .

3.2.10 异乳糖(1)的制备

将化合物 14 (14.1 g, $5 \mathrm{mmol}$ )溶于 $300 \mathrm{~mL}$ 90\%三氟 
乙酸水溶液中, 室温下反应, TLC $[V$ (石油醚)： $V($ 乙酸 乙酯 $)=1: 1$ 追踪检测至反应完全，减压浓缩，用 20 $\mathrm{mL} \times 3$ 甲苯共蒸除尽溶剂. 将浓缩物溶于 $60 \mathrm{~mL}$ 甲醇, 向体系中滴加新制甲醇钠溶液, 调体系 $\mathrm{pH}$ 值 $\approx 10$, 室 温下反应, TLC $[V$ (石油醚) $: V($ 乙酸乙酯 $)=1 ： 2]$ 追踪检 测至反应完全. 向体系中加入酸性离子树脂调节体系 $\mathrm{pH}$ 至中性, 抽滤除去树脂, 滤液浓缩至剩余约 $15 \mathrm{~mL}$ 溶 剂, 趁热加入 $30 \mathrm{~mL}$ 无水乙醇、 $6 \mathrm{~mL}$ 水, 冷冻放置析出 白色粉末状固体 1 (4.5 g, 收率 90\%). [ $\alpha]_{\mathrm{D}}^{25}+67(c$ $\left.0.029, \mathrm{H}_{2} \mathrm{O}\right) ;{ }^{13} \mathrm{C} \mathrm{NMR}\left(75 \mathrm{MHz}, \mathrm{CDCl}_{3}\right) \delta: 105.38,98.07$, 94.23, 76.99, 76.14, 73.52, 72.88, 72.57, 71.61, 70.80, 63.09, 51.00; HRMS (ESI) calcd for $\mathrm{C}_{12} \mathrm{H}_{22} \mathrm{O}_{11}(\mathrm{M}+$ $\left.\mathrm{NH}_{4}\right)^{+} 360.15004$, found 360.15051 [文献数据 ${ }^{[21]}{ }^{13} \mathrm{C}$ NMR (100 MHz, D $2 \mathrm{O}) \delta$ : 106.1 (C-1'), 98.8 (C-1 $\beta), 94.9$

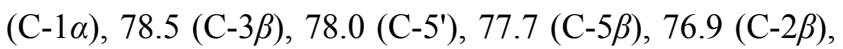
75.5 (C-3' and C-3 $\alpha$ ), 74.2 (C-2 $\alpha), 73.6$ (C-2'), 73.3 (C-5 $\alpha$ ),

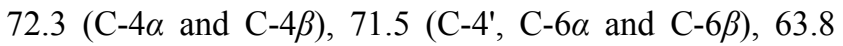
$\left.\left(\mathrm{C}-6^{\prime}\right)\right]$.

\section{References}

[1] Jobe, A.; Bourgeois, S. J. Mol. Biol. 1972, 69, 397.

[2] Jacob, F.; Monod, J. J. Mol. Biol. 1961, 3, 318.

[3] Huber, R. E.; Wallenfels, K.; Kurz, G. Can. J. Biochem. 1975, 53, 1035.

[4] Prosperi, D.; Panza, L.; Haltrich, D.; Nonini, M.; Riva, S. J. Carbohydr. Chem. 2003, 22, 267.

[5] Pazur, J. H.; Tipton, C. L.; Budovich, T.; Marsh, J. M. J. Am. Chem.
Soc. 1958, 80, 119

[6] Magnusson, G.; Nilsson, U. J. Glycoscience 2001, 2, 1543.

[7] Kong, F. Curr. Org. Chem. 2003, 7, 841.

[8] Malik, S.; Sharma, A.; Kartha, K. P. R. Trends Carbohydr. Res. 2009, 1,36 .

[9] Ma, Z.; Zhang, J.; Kong, F. Carbohydr. Res. 2004, 339, 1761.

[10] Zhang, J.; Liang, X.; Wang, D.; Kong, F. Carbohydr. Res. 2007, 342, 797.

[11] Deulofeu, V.; Deferrari, J. O. J. Org. Chem. 1952, 17, 1097.

[12] Barker, G. R. Methods Carbohydr. Chem. 1963, 2, 168.

[13] Schmidt, R. R. Angew. Chem. 1986, 98, 213.

[14] [Zhang, J.; Kong, F. Tetrahedron 2003, 59, 1429.

[15] Zong, G.; Yan, S.; Liang, X.; Wang, D.; Zhang, J. Chin. J. Org. Chem. 2011, 31, 2126 (in Chinese)

(宗广辉, 颜世强, 梁晓梅, 王道全, 张建军, 有机化学, 2011, 31, 2126.)

[16] Zong, G. H.; Yan, S. Q.; Liang, X. M.; Zhang, J. J.; Wang, D. Q.; Kong, F. Z. Chin. Chem. Lett. 2009, 20, 127.

[17] Cai, X.; Zong, G.; Xu, Y.; Zhang, J.; Liang, X.; Wang, D. Carbohydr. Res. 2010, 345, 1230.

[18] Zong, G.; Feng, Y.; Liang, X.; Chen, L.; Zhang, J.; Wang, D. Carbohydr. Res. 2010, 345, 2067.

[19] Zong, G.; Yu, N.; Xu, Y.; Zhang, J.; Wang, D.; Liang, X. Synthesis 2010, 1666.

[20] Zong, G.; Cai, X.; Liang, X.; Zhang, J.; Wang, D. Carbohydr. Res. 2011, 346, 2533.

[21] Suyama, K.; Adachi, S.; Toba, T.; Sohma, T.; Hwang, C.; Itoh, T. Agric. Biol. Chem. 1986, 50, 2069.

[22] Rio, S.; Beau, J. M.; Jacquinet, J. C. Carbohydr. Res. 1991, 219, 71.

[23] Zhang, L.; Zhang, W.; Feng, Y.;Yu, X.; Meng, X. Chem. Ind. Eng. 2004, 21, 414 (in Chinese).

(张利梅; 张卫红; 冯亚青; 于晓佳; 孟祥启, 化学工业与工程, $\mathbf{2 0 0 4}, 21,414$.)

[24] Tsui, H.-C.; Paquette, L. A. J. Org. Chem. 1998, 63, 9968.

(Cheng, F.) 